\title{
An analysis of double exposure lithography options
}

\author{
Saul Lee ${ }^{a}$, Je rey Byers ${ }^{b}$, Kane Jen ${ }^{a}$, Paul Zimmerman ${ }^{b}$, Bryan Rice ${ }^{b}$, Nicholas J. Turro ${ }^{c}$, and \\ C. Grant Willson ${ }^{a}$ \\ ${ }^{a}$ Department of Chemical Engineering, The University of Texas at Austin, Austin, Texas, USA; \\ ${ }^{b}$ SEMATECH, 2706 Montopolis Drive, Austin, Texas, USA; \\ ${ }^{c}$ Department of Chemistry, Columbia University, New York, New York, USA
}

\begin{abstract}
The current optical photolithography technology is approaching the physical barrier to the minimum achievable feature size. To produce smaller devices, new resolution enhancement technologies must be developed. Double exposure lithography has shown promise as potential pathway that is attractive because it is much cheaper than double patterning lithography and it can be deployed on existing imaging tools. However, this technology is not possible without the development of new materials with nonlinear response to exposure dose. The performance of existing materials such as reversible contrast enhancement layers (rCELs) and theoretical materials such as intermediate state two-photon (ISTP) and optical threshold layer (OTL) materials in double exposure applications was investigated through computer simulation. All three materials yielded process windows in double exposure mode. OTL materials showed the largest process window (DOF $0.137 \mu \mathrm{m}$, EL $5.06 \%$ ). ISTP materials had the next largest process window (DOF $0.124 \mu \mathrm{m}$, EL $3.22 \%$ ) followed by the rCEL $(0.105 \mu \mathrm{m}, 0.58 \%$ ). This study is an analysis of the feasibility of using the materials in double exposure mode.
\end{abstract}

Keywords: Double Exposure Lithography, Immersion Lithography, Double Patterning Lithography, Resolution Enhancement Techniques

\section{INTRODUCTION}

The current technological progression of the photolithography industry has reached a limit in the maximum achievable resolution. Resolution as determined by the half pitch critical dimension (CD) is limited by the Rayleigh equation

$$
C D=\frac{k_{1}}{N A}
$$

where $k_{1}$ is the process aggressiveness factor, is the wavelength of the imaging tool, and $N A$ is the numerical aperture of the imaging lens. To reduce the half pitch $C D$, the industry must reduce $k_{1}$ or , or increase $N A$. The theoretical minimum value for $k_{1}$ with single exposure is 0.25 , but the generally accepted manufacturability limit is 0.27 . The current industry standard imaging tool has a wavelength of $193 \mathrm{~nm}$. Future imaging tools are proposed to operate in the Extreme Ultra Violet (EUV) range with a of $13.4 \mathrm{~nm}$, however, the EUV technology will most likely not be viable until after 2013. With water immersion lithography, the maximum achievable $N A$ is approximately 1.35. Increasing the $N A$ requires simultaneous development of a high index lens material along with high index fluids and high index resists. Without major breakthroughs in optical materials, $N A$ will plateau near 1.35. Given these parameters, the current $C D$ limit is approximately $38 \mathrm{~nm}$ half pitch.

To enable lithography at sub $38 \mathrm{~nm}$ half pitch, the industry will need to consider alternative resolution enhancement technologies. Two exposure passes have been proposed as a possible resolution enhancement technique for existing photolithography imaging systems. A single mask with high feature density that is di cult to resolve can be split into two exposures each with lower feature density that can be easily resolved. When combined, the two exposures replicate the original mask.

Further author information: Send correspondence to Professor C. Grant Willson Mail: 1 University Station C0400, Austin, TX, 78712

E-mail: willson@che.utexas.edu

Optical Microlithography XXI, edited by Harry J. Levinson, Mircea V. Dusa,

Proc. of SPIE Vol. 6924, 69242A, (2008) $\cdot 0277-786$ X/08/\$18 $\cdot$ doi: 10.1117/12.773030

Proc. of SPIE Vol. 6924 69242A-1 


\subsection{Double Exposure Lithography versus Double Patterning Lithography}

Double exposure lithography (DEL) and double patterning lithography (DPL) are proposed approaches to performing the two exposure passes. DEL is defined as a two exposure pass lithographic process that does not require the removal of the wafer from the exposure tool chuck between passes. DPL is defined as a two exposure pass lithographic process that requires a chemical development of the photoresist layers and possibly an intermediate etch step. The DPL processing approaches will require the removal of the wafer from the exposure tool chuck and loss of overlay registration. DEL and DPL processes are illustrated in Figure 1. The benefits of

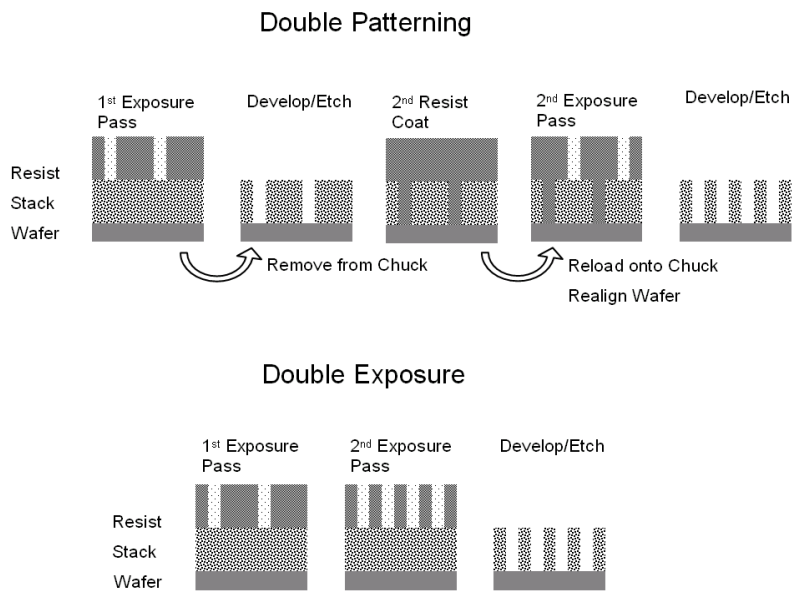

Figure 1. Comparison of the double patterning lithography(development/etch scheming shown) and double exposure lithography processes.

DEL and DPL principally include the ability to use existing exposure tools to print technology nodes below the $N A$ limit for single exposure processes. This could mean a lower cost of ownership as these techniques can in principle be deployed without costly capital investment. However, the two exposure passes require doubling the number of masks and reduced throughput due to increased processing time. The process time is dramatically increased in the DPL process because of the additional process steps compared to the DEL process. In addition, the removal of the wafer from the wafer chuck between exposures poses severe overlay issues that may be di cult to overcome, especially at the CDs where this technology will be implemented. The DEL process only introduces an additional exposure pass, and since the wafer is not removed from the imaging tool between exposures, the overlay issues are minimized. The reduced cost of ownership of DEL suggests that it would be the preferred technique.

\section{2 "Resist Memory" E ect}

The DEL infrastructure is currently available on existing state of the art exposure tools. However, imaging below a $k_{1}$ value of 0.25 with double exposure is impossible without the development of new materials. Conventional resists have a "memory" e ect that prevents proper replication of the mask image. That is, sub-threshold exposure in the first exposure pass reduces the dose required to render the resist soluble in the second exposure pass. For example, the normalized aerial image intensities for the first exposure pass reaching the resist of equal lines and spaces can be described by the following

$$
I_{P \text { ass } 1}=A \cos ^{2} \frac{\pi \cdot x}{p i t c h}+B
$$

where $A$ is a constant describing the amplitude and $B$ is the minimum image intensity. For the second exposure pass, the mask and, consequently, the aerial image are translated by half pitch and lead to the following intensity function

$$
I_{P \text { ass } 2}=A \cos ^{2} \frac{\pi \cdot x}{p i t c h}+\frac{\pi}{2}+B=A \sin ^{2} \frac{\pi \cdot x}{p i t c h}+B
$$


The photochemical response of the resist results in a linear summation of the absorbed intensities from the two exposure pass. This leads to the following intensity function within the resist

$$
I_{\text {Sum }}=I_{\text {ass } 1}+I_{\text {Pass } 2}=A \cos ^{2} \frac{\pi \cdot x}{\text { pitch }}+A \sin ^{2} \frac{\pi \cdot x}{\text { pitch }}+2 B=A+2 B=\text { a Constant } !
$$

Consequently, the two individual mask images are not resolved when double exposed. This concept is illustrated in Figure 2.

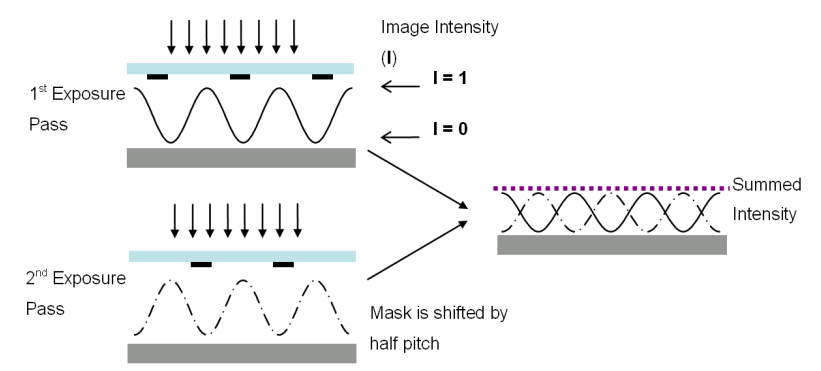

Figure 2. Summation of the intensity of two exposure passes and the e ect of dose reciprocity.

The resist system converts the separate light images, intensity versus position, into chemical images, chemical composition versus position. Mathematically, this conversion of the light image into a chemical image can be represented by a translation function $f(I)$. In the case of standard resist systems, this translation function has the linear addition property

$$
f\left(I_{P \text { ass } 1}+I_{P \text { ass } 2}\right)=f\left(I_{P \text { ass } 1}\right)+f\left(I_{P \text { ass } 2}\right) .
$$

Resolving the mask features requires a material with a nonlinear response to exposure such that

$$
f\left(I_{P \text { ass } 1}+I_{P \text { ass } 2}\right)=f\left(I_{P \text { ass } 1}\right)+f\left(I_{P \text { ass } 2}\right)
$$

and the "resist memory" behavior is minimized.

\subsection{Potential DEL Materials}

Several materials have been proposed to implement a nonlinear response to exposure and theoretically permit double exposure pitch doubling including contrast enhancement layers (CELs), two-photon materials, intermediate state two-photon (ISTP) materials, and optical threshold layers (OTLs). These materials and their theory of operation are described in the following sections.

\subsubsection{Contrast Enhancement Layer}

Contrast enhancement layers (CELs) are strongly absorbing materials that increase transparency, or photobleach, ${ }^{1}$ when exposed to light. A CEL is normally applied directly on top of the resist layer. During exposure, energy is first devoted to photobleaching the CEL. As the CEL becomes transparent, the energy is then able to reach the resist and initiate the solubility switch. Light can only penetrate through the CEL in regions where aerial image intensities are high (non-opaque regions on the mask) and cannot reach the resist in regions where aerial image intensities are lower (opaque regions on the mask). This introduces a nonlinear transfer of the applied aerial image into the photoresist and improves the resolution. CELs can be divided into two di erent subtypes, namely, reversible (rCEL) and irreversible (irCEL). The main di erence between the two subtypes is that, in rCELs, the photobleached regions can return to the initial opaque state between exposure passes whereas in irCELs, the photobleaching is irreversible. Details on the existing chemistries and transmission characteristics for CELs have been described in previously published work ${ }^{2-6}$ and are not discussed here. 


\subsubsection{Two-Photon Materials}

Two-photon photoresist systems involve the incorporation of photoacid generators (PAGs) that require the simultaneous absorption of two photons to induce the photochemical acid generation. The chemical reaction for a two-photon photoacid generator can be described by the following reaction

$$
\mathrm{PAG}+I(h)+I(h) \text { GGGGA Acid, }
$$

where is the quantum e ciency of the two-photon photochemical reaction. Since the simultaneous absorption of two photons is required for the reaction, the probability of conversion is proportional to the light intensity squared, which provides a nonlinear response to exposure energy

$$
f(I) \approx I \cdot I
$$

and the DEL conversion is

$$
f\left(I_{P \text { ass } 1}\right)+f\left(I_{P \text { ass } 2}\right) \approx I_{P \text { ass } 1} \cdot I_{P \text { ass } 1}+I_{P \text { ass } 2} \cdot I_{P \text { ass } 2}=f\left(I_{P \text { ass } 1}+I_{P \text { ass } 2}\right) .
$$

Unlike the CEL, two-photon materials are not enhancement layers that are applied on top of existing resists, but rather the nonlinear response is incorporated directly into the resist formulation. This eliminates complexities introduced by the addition of an extra film layer such as depth of focus and material compatibility.

Two-photon resist systems for microfabrication using laser writing systems have been reported previously. ${ }^{7}$ These systems employ specially designed PAGs with high two-photon absorbance cross sections. High e ciency two-photon PAGs have not yet been developed to work with $193 \mathrm{~nm}$. Analysis (to be presented in future work) of the two-photon reaction kinetics suggests that a very large increase in exposure source output would be required to produce a viable level of process throughput. As such, the two-photon material type was not included in the simulation studies.

\subsubsection{Intermediate State Two-Photon Materials}

Intermediate state two-photon (ISTP) layers are materials that generate acid molecules in a reversible two step process. Similar to two-photon materials, ISTP materials alter the acid generation behavior of the resist medium. Although each step requires the absorption of a photon, ISTP materials are not true two-photon processes in that the acid production does not have a quadratic dependence on dose. The reaction sequence is

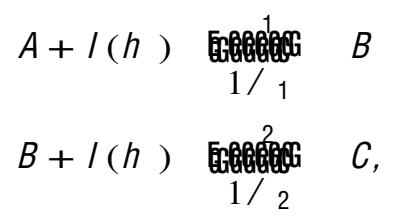

where 1 and 2 represent the cross-sections and $1_{1}$ and 2 represent the lifetimes of the species.

Although ISTP materials do not exhibit true two-photon behavior, they may require significantly lower doses to generate acid compared to two-photon resists. The trade o between lower reaction orders may be o set by the lower doses. The behavior of ISTP materials depends on the ability of the intermediate species to revert to the initial state. A build up of the intermediate species will e ectively render the sequence to become a first ordered reaction that is controlled by a rate limiting step. Therefore, the characteristics of the exposing laser such as the energy per pulse $(A 0)$, pulse cycle time ( $t_{f}$, also the inverse of the repetition rate), and full width half max (FWHM) also have to be considered. 


\subsubsection{Optical Threshold Materials}

Optical threshold layers (OTLs) are materials that require the absorption of a threshold exposure dose to induce a photochemical event. The exposure threshold gives the material a region of nonlinear response to exposure dose and allows OTLs to be used as double exposure resists. Nonlinearity derives from the fact that any dose absorbed below the threshold does not cause reactions to occur.

Above the dose threshold, a step change in conversion occurs and is described by the following piecewise function

$$
f(I)=\begin{array}{cl}
{[P A C]_{t h}} & , \quad E_{\text {Actual }} \geq E_{t h} \\
1 & , \quad E_{\text {Actual }}<E_{t h}
\end{array} \text {, where } E_{\text {Actual }}=\underset{\text { pulses }}{l} l \cdot d t,
$$

where $E_{\text {Actual }}$ and $E_{t h}$ represent the actual dose received by the material and the threshold dose, respectively. $[P A C]_{t h}$ is the step wise conversion concentration of the photoactive compound after reaching $E_{t h}$. Equation 11 shows the behavior of an ideal OTL material.

Analogous thermal resist systems are already in use in the printing industry. ${ }^{8}$ Thermal resists rely upon a thermal image instead of an optical image. These systems can, for example, function by exploitation of a phase change that is inherently threshold-like. If a material is heated to a temperature just below its melting point and then cooled, it does not "remember" the previously applied thermal dose. Chapman et al. have investigated inorganic thermal resist systems ${ }^{9}$ that use $\mathrm{Bi} / \mathrm{In}$ bilayers as an etch masking layer for silicon. However, the use of $\mathrm{Bi}$ and In metals are not compatible with the photolithography process as the target semiconductor devices are very susceptible to metal contamination. Chemical systems with similar properties for optical images have to be developed to use this technology with lithographic imaging systems.

\subsection{Feasibility Studies}

These materials are not the only possibilities, but they do provide a reasonable range of resist designs to explore the feasibility of DEL as a technology choice. Of the proposed materials, only CELs and two-photon materials have been extensively studied and have established chemical systems. ISTP and OTL materials do not currently exist for use in semiconductor applications. However, their theoretical mechanisms are considered to test their viability as a possible DEL candidates. This work investigates the feasibility of the materials for use in DEL applications through simulation and is meant to guide our materials development e ort.

\section{SIMULATION CONDITIONS}

The performance of the di erent material types in double exposure mode and the dependence on their material properties were evaluated by computer simulation using a combination of PROLITH lithography simulator and custom code. In all cases, the optical imaging portion was performed with PROLITH. The material responses of the reversible CEL was studied using the PROLITH simulator. However, commercial models for the ISTP and OTL materials do not yet exist as the materials are not currently used in production, so a custom simulator was developed to model the material behaviors in these systems.

\subsection{Imaging Setup}

A half pitch CD of $25 \mathrm{~nm}$ was targeted using a 1.2 NA water immersion exposure system in double exposure mode. This is an e ective $k_{1}$ of 0.155 . An azimuthally polarized cross-quadrupole with center $=0.8$ and radius $=0.15$ was used as the illuminator. Di erent masks were used for each of the two exposure passes. The masks were 50 $\mathrm{nm}$ line/space phase shift masks with $6 \%$ attenuation. The two masks were o set by $50 \mathrm{~nm}$ between exposure passes. As described previously, ${ }^{2}$ DEL with positive tone resists is a trench-based process as opposed to the line-based process expected with a single exposure pass. Consequently, the target line is expected to form at the interface of the opaque and bright regions as opposed to the center of the bright regions. A focus-exposure matrix was run for each material system, and the resulting $C D$ was observed. 
Table 1. Parameters used for the base resist system.

\begin{tabular}{|c|c|}
\hline Property & Value \\
\hline $\mathrm{n}$ & 1.70 \\
\hline Dill A $\left(\mu \mathrm{m}^{-1}\right)$ & 0 \\
\hline Dill B $\left(\mu \mathrm{m}^{-1}\right)$ & 1.47 \\
\hline Dill C $\left(\mathrm{cm}^{2} / \mathrm{mJ}\right)$ & 0.0478 \\
\hline$[\mathrm{Q}] /[\mathrm{PAG}]$ & 0.2 \\
\hline$M_{t h}$ & 0.75 \\
\hline $\mathrm{D}_{\mathrm{H}}\left(\mathrm{nm}^{2} / \mathrm{s}\right)$ & 0.223 \\
\hline $\mathrm{D}_{Q}\left(\mathrm{~nm}^{2} / \mathrm{s}\right)$ & 0.0 \\
\hline $\mathrm{k}_{\mathrm{a}}(1 / \mathrm{s})$ & 0.100 \\
\hline $\mathrm{k}_{\mathrm{b}}(1 / \mathrm{s})$ & $4.85 \times 10^{8}$ \\
\hline
\end{tabular}

\subsection{Film Stack and Base Resist System}

The film stack consisted of $50 \mathrm{~nm}$ of resist on $31 \mathrm{~nm}$ of a single layer of bottom anti-reflective coating (BARC) $(\mathrm{n}=1.82, \mathrm{k}=0.46)$. The substrate was silicon with a $2 \mathrm{~nm}$ layer of $\mathrm{SiO}_{2}$. The resist simulations were based upon a typical $193 \mathrm{~nm}$ resist system. The base resist parameters are shown in Table 1. In the case of the OTL and ISTP materials, the acid generation behavior di ers from the base resist and was described with custom models.

\subsection{Reversible CEL Simulation Parameters}

To study the CEL behavior, a $50 \mathrm{~nm}$ CEL was applied on top of the film stack. The Dill A parameter was varied from $10 \mu \mathrm{m}^{-1}$ to $50 \mu \mathrm{m}^{-1}$. The CEL parameters are shown in Table 2. Since a reversible CEL system provides

Table 2. Simulation parameters used for the reversible CEL material.

\begin{tabular}{|c|c|}
\hline Parameter & Value \\
\hline $\mathrm{n}$ & 1.69 \\
\hline Dill $\mathrm{A} \mu \mathrm{m}^{-1}$ & $0-50$ \\
\hline Dill B $\mu \mathrm{m}^{-1}$ & 0 \\
\hline Dill $\mathrm{C}\left(\mathrm{cm}^{2} / \mathrm{mJ}\right)$ & 0.11 \\
\hline
\end{tabular}

better performance than an irreversible system, the rCEL was selected for the study.

\subsection{ISTP Simulation Parameters}

To study the performance of the ISTP material, the acid generation behavior described in the previous section was solved using a 4 th order Runge-Kutta method. ${ }^{10}$ The parameters and values used in the simulation are listed in Table 3. To simplify the calculations, 2 was assumed to be very large such that the conversion of the intermediate species to acid is assumed to be irreversible.

\subsection{OTL Simulation Parameters}

The OTL acid generation behavior described in the previous section was implemented. A dose threshold, $E_{t h}$, of $10 \mathrm{~mJ} / \mathrm{cm}^{2}$ was used with a threshold photoacid compound conversion, $[P A C]_{t h}$, value of 0.75 . 
Table 3. Simulation parameters used for the ISTP material.

\begin{tabular}{|c|c|}
\hline Parameter & Value \\
\hline${ }_{1}\left(\mathrm{~cm}^{2} / \mathrm{mJ}\right)$ & 0.235 \\
\hline $2\left(\mathrm{~cm}^{2} / \mathrm{mJ}\right)$ & 0.235 \\
\hline $1(\mathrm{~s})$ & 0.006 \\
\hline$t_{f}(\mathrm{~s})$ & 0.00025 \\
\hline$A 0\left(\mathrm{~mJ} / \mathrm{cm}^{2}\right)$ & 0.05 \\
\hline & 1 \\
\hline FWHM $(\mathrm{ns})$ & 20 \\
\hline
\end{tabular}

\section{RESULTS AND DISCUSSION}

All three materials yielded nonlinear resist response when used in double exposure mode. The resist profiles are shown in Figure 3 to Figure 5 and summarized in Table 4.
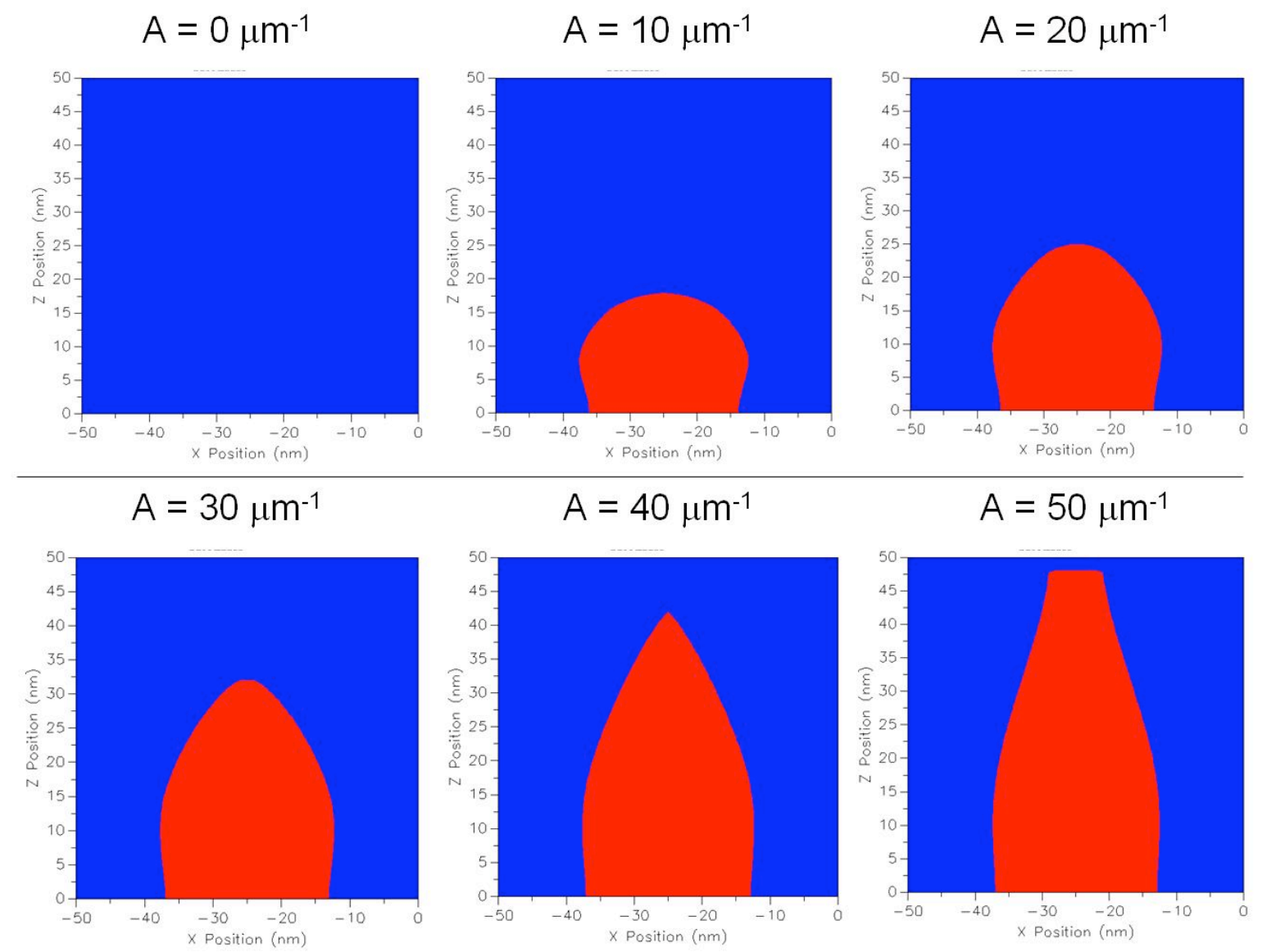

Figure 3. Resist profile of rCEL with varying Dill A parameter.

The simulation results from the focus-exposure experiments were analyzed using ProDATA to generate simulated Bossung plots and exposure latitude (EL) versus depth of focus (DOF) plots. The results are shown in 


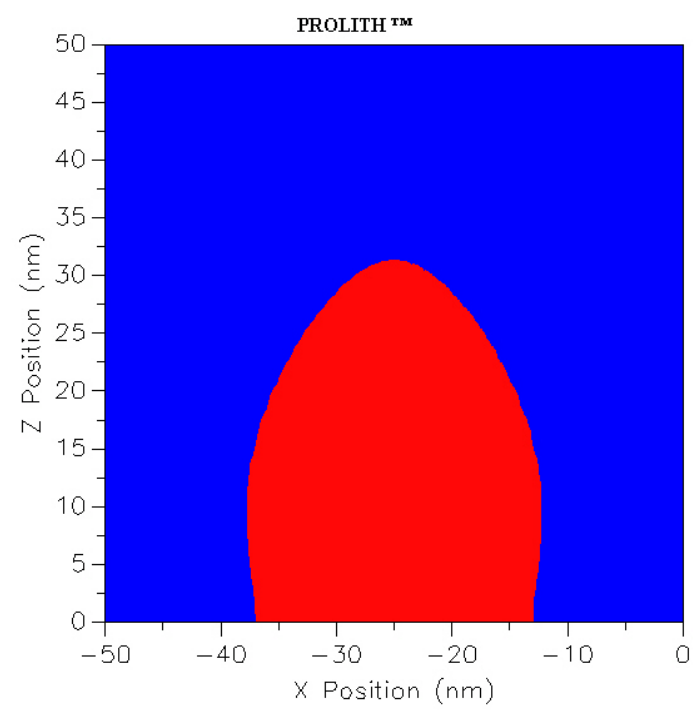

Figure 4. Resist Profile for ISTP material.

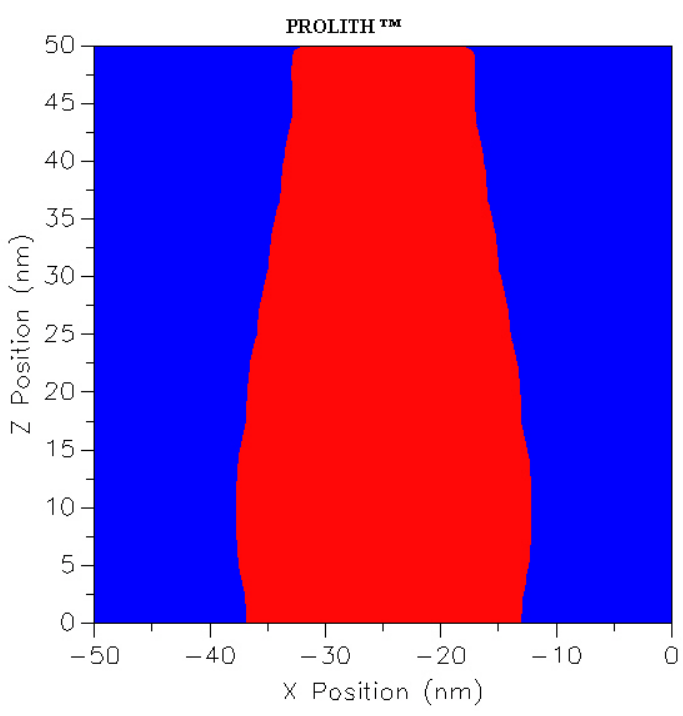

Figure 5. Resist Profile for OTL material.

Figure 6 to Figure 8 and are summarized in Table 5. Simulated EL versus DOF plots with respect to the di erent Dill A parameters are shown in Figure 9.

It is important to note that $\mathrm{CD}$ was the only output metric considered in the process window calculations. In most manufacturing environments, other parameters such as sidewall angle and resist loss would also need to be optimized to produce functional devices. However, the main goal of this work is to demonstrate the proof of concept of the theoretical materials. Optimization of such parameters is beyond the scope of this work.

Figure 3 shows the e ects of the Dill A parameter on the resist profile. For the test case of a Dill A parameter of $0 \mu \mathrm{m}^{-1}$, no resist profile was observed. This finding is consistent with the behavior of conventional resists since no nonlinearity was applied and further verified that the DEL process does not provide resolved images in conventional resists.

In all cases, increasing the Dill A parameter decreased resist loss and improved the shape of the resulting image. Figure 9 shows that the process window was also widened with the increase. Increasing the Dill A param- 
Critical Dimension vs. Focus/Exposure

for rCELA = $20 \mathrm{um}^{\wedge}-1$

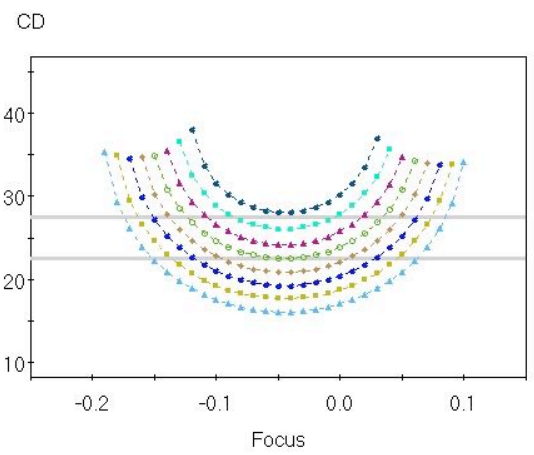

Exposure Latitude vs. DOF

for rCELA = 20 um -1

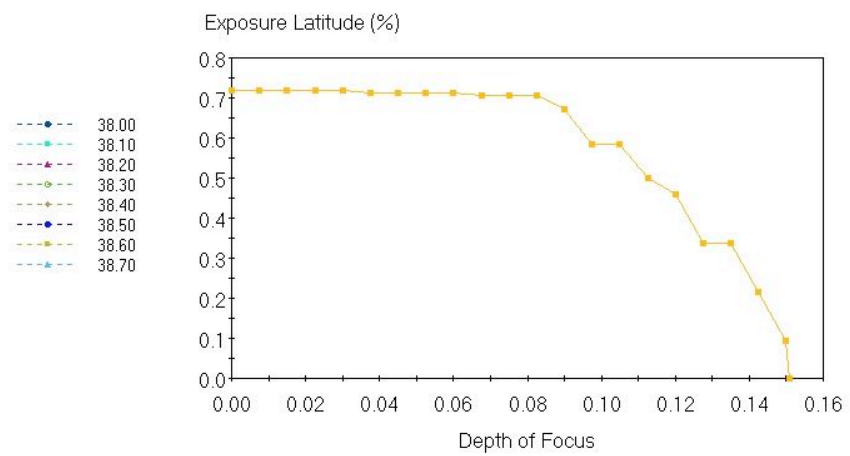

Figure 6. Simulated Bossung plot and EL versus DOF for rCEL material.

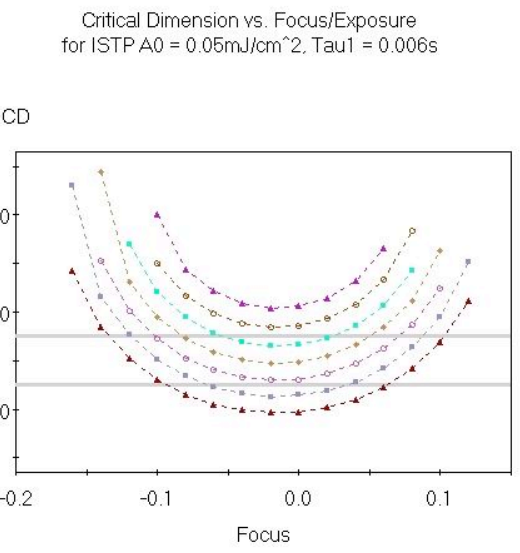

Figure 7. Simulated Bossung plot and EL versus DOF for ISTP material.

Critical Dimension vs. Focus/Exposure for OTL Eth $=10 \mathrm{~mJ} / \mathrm{cm}^{\wedge} 2$, PACth $=0.75$

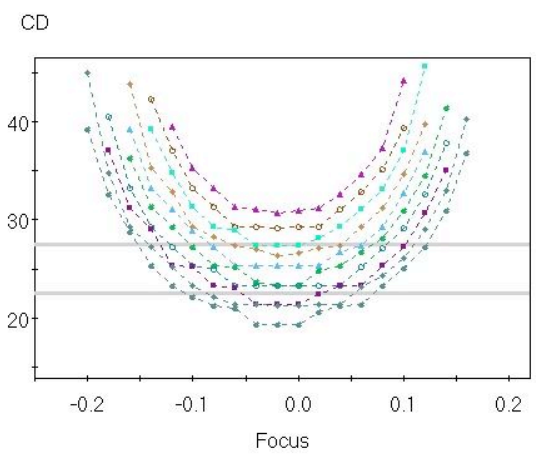

Exposure Latitude vs. DOF for ISTPA0 $=0.05 \mathrm{~mJ} / \mathrm{cm}^{\wedge} 2$, Tau $1=0.006 \mathrm{~s}$

Exposure Latitude (\%)

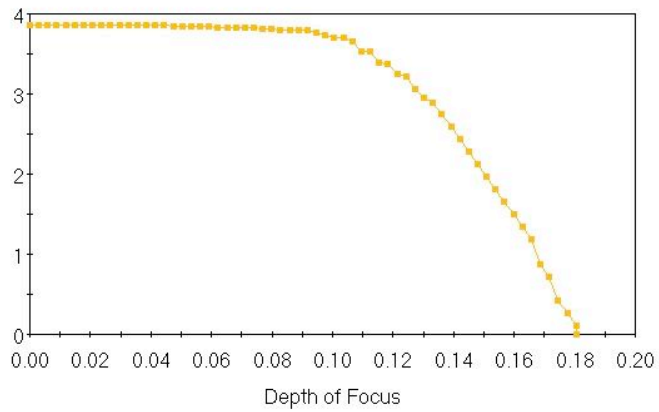

Figure 8. Simulated Bossung plot and EL versus DOF for OTL material. 
Table 4. Summary of resist profile metrology.

\begin{tabular}{|c|c|c|c|}
\hline Material & $\begin{array}{c}\text { Dose } \\
\left(\mathrm{mJ} / \mathrm{cm}^{2}\right)\end{array}$ & $\begin{array}{c}\text { Sidewall } \\
\text { Angle }\left({ }^{0}\right)\end{array}$ & $\begin{array}{c}\text { Resist } \\
\text { Loss }(\mathrm{nm})\end{array}$ \\
\hline rCEL $\mathrm{A}=10 \mu \mathrm{m}^{-1}$ & 24.6 & 74.9 & 32.2 \\
\hline rCEL A $=20 \mu \mathrm{m}^{-1}$ & 38.2 & 74.4 & 25.0 \\
\hline rCEL A $=30 \mu \mathrm{m}^{-1}$ & 56.4 & 71.1 & 18.0 \\
\hline rCEL A $=40 \mu \mathrm{m}^{-1}$ & 79.6 & 70.2 & 8.1 \\
\hline rCEL A $=50 \mu \mathrm{m}^{-1}$ & 108 & 74.8 & 2.0 \\
\hline ISTP & 36.5 & 71.9 & 18.8 \\
\hline OTL & 39.3 & 81.9 & 0.2 \\
\hline
\end{tabular}

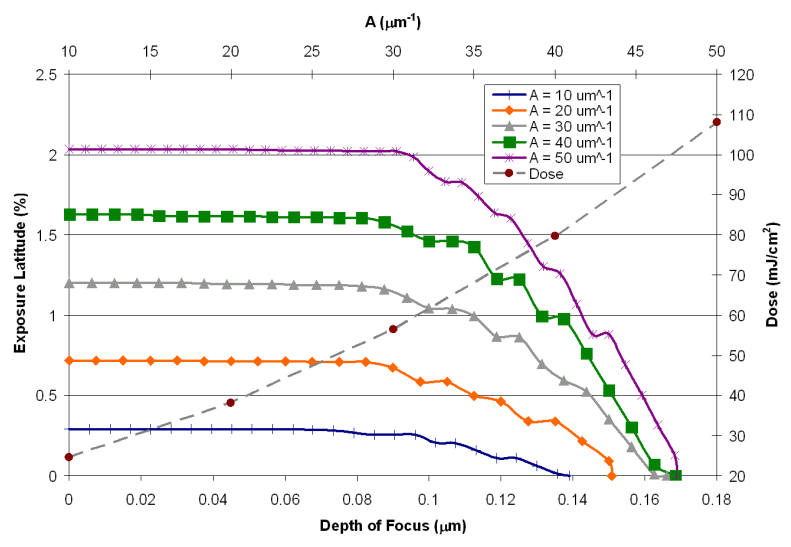

Figure 9. Process windows of rCEL materials varying the Dill A parameter.

eter showed improvement for EL. However, only marginal improvement was observed for the DOF. Increasing the Dill A parameter also led to increases in the dose requirement. An increase in the Dill A parameter from 10 $\mu \mathrm{m}^{-1}$ to $50 \mu \mathrm{m}^{-1}$ required approximately 4.4 fold increase in dose. Another factor to consider is the feasibility of obtaining rCEL materials with high Dill A values. Without increasing the Dill A parameter, it is theoretically possible to increase the absorbance of the rCEL layer by increasing the layer thickness. However, the oblique incident angles resulting from operating at $N A$ values greater than 1 may lead to loss in depth of focus if the layer becomes too thick. rCEL materials showed nonlinear behavior in DEL mode, however, image quality and process window improvement was only observed for rCELs with very high Dill A parameters $\left(>30 \mu \mathrm{m}^{-1}\right)$. Even if physical analogs with such high Dill A parameters are obtainable, the improvements are marginal and come at the cost of large dose increases.

Table 4 shows that an rCEL with Dill A parameter of $20 \mu \mathrm{m}^{-1}$ has a comparable dose requirement to that of ISTP and OTL materials. Results from this run were used for subsequent comparisons with ISTP and OTL materials.

Figure 4 shows the resist profile of the ISTP material. The profile is comparable to rCEL having slightly lower sidewall angle, $71.9^{\circ}$, and reduced resist loss, $18.8 \mathrm{~nm}$. Table 5 shows that ISTP has a larger process window than rCEL. The parameters of interest a ecting the nonlinear acid generation behavior of the material are the energy delivered per pulse, A0, and the reversible rate constant of the intermediate state, $1 /{ }_{1}$. For a given set of laser parameters, large values of A0 or 1 lead to faster conversion of PAC thus reducing the required exposure dose. However, the dose reduction also leads to a decrease in nonlinearity. Since the laser can only deliver integer numbers of pulses, the magnitude of $\mathrm{A} 0$ has to be within a manageable increment such that small 
Table 5. Summary of process windows for the rCEL, ISTP, and OTL materials.

\begin{tabular}{|c|c|c|}
\hline Material & Depth of Focus $(\mu \mathrm{m})$ & Exposure Latitude $(\%)$ \\
\hline rCEL & 0.105 & 0.58 \\
\hline ISTP & 0.124 & 3.22 \\
\hline OTL & 0.137 & 5.06 \\
\hline
\end{tabular}

deviations in the pulse delivery will not drastically a ect the CD. The parameters had to be optimized so that the system will retain nonlinear behavior but at the same time yield features within reasonable exposure dose ranges. ISTP materials showed a larger process window and improved resist profile than rCEL, and could be a potential DEL material provided that materials with the specified kinetics and time constants can be identified.

Figure 5 shows the resist profile of the OTL material. The profile shows a significant reduction in resist loss compared to both rCEL and ISTP resist profiles and slight improvement in the sidewall angle. The OTL material also has the largest process window of the three materials investigated. The threshold dose requirement behavior of the OTL material served e ectively to filter out regions of low intensity. In addition, the threshold conversion response of the PAC resulted in improved image contrast. Because no such physical systems exist, the threshold dose, $E_{t h}$, and PAC conversion, $[P A C]_{t h}$, were chosen such that they would provide a defined solubility switch within comparable dose ranges. Performance in physical systems may di er depending on the thresholding mechanism. OTL materials showed the best performance (i.e. largest process window and best resist profile) compared to rCEL and ISTP materials and would be suitable for DEL applications. This suggests that potential mechanisms, either chemical or physical, need to be explored.

\section{CONCLUSIONS}

DEL o ers several advantages over DPL, but it requires new materials with nonlinear dose response. We have employed simulations to explore several potential DEL material options. The modeling results show that twophoton materials will not be feasible unless achievable laser peak power in exposure tools can be significantly increased. rCEL materials demonstrated nonlinear behavior in DEL mode, however, image quality and process window improvement was only observed for rCELs with very high Dill A parameters $\left(>30 \mu \mathrm{m}^{-1}\right)$. Even if physical analogs with such high Dill A parameters are obtainable, the improvements are marginal. ISTP materials showed a larger process window than rCEL. The challenges with this approach are identifying materials with the specified kinetics and the ability to tune the time constants. OTL materials showed the best performance with the largest process window and best resist profile. There are no physically functional optical analogs with the thresholding behavior. Potential mechanisms, either chemical or physical, need to be explored. From our feasibility studies, we believe that the ISTP and OTL materials have the greatest potential for use in DEL applications and warrant our investment in materials development.

\section{ACKNOWLEDGMENTS}

The authors would like to thank SEMATECH for financial support of this project, KLA-Tencor for the donations of PROLITH and ProDATA licenses, Mark Smith and Trey Graves of KLA-Tencor, and Intel Corporation for the donations of simulation machines.

\section{Disclaimers}

SEMATECH, and the SEMATECH logo are registered servicemarks of SEMATECH, Inc. All other servicemarks and trademarks are the property of their respective owners. 


\section{REFERENCES}

[1] Gri ng, B. F. and West, P. R., "Contrast enhanced lithography," Solid State Technology 28(5), 152-7 (1985).

[2] Byers, J., Lee, S., Jen, K., Zimmerman, P., Turro, N. J., and Willson, C. G., "Double exposure materials: Simulation study of feasibility," Journal of Photopolymer Science and Technology 20(5), 707-717 (2007).

[3] Krayushkin, M. M., Uzhinov, B. M., Martynkin, A. Y., Dzhavadov, D. L., Kalik, M. A., Ivanov, V. L., Stoyanovich, F. M., Uzhinova, L. D., and Zolotarskaya, O. Y., "Thermally irreversible photochromic dithienylethenes," International Journal of Photoenergy 1(3), 183-190 (1999).

[4] Becker, R. S. and Michl, J., "Photochromism of synthetic and naturally occurring $2 \mathrm{H}$-chromenes and $2 \mathrm{H}-$ pyrans," Journal of the American Chemical Society 88(24), 5931-3 (1966).

[5] Grant, B. D., Clecak, N. J., Twieg, R. J., and Willson, C. G., "Deep UV photoresists I. Meldrum's diazo sensitizer," IEEE Transactions on Electron Devices 28(11), 1300-1305 (1981).

[6] West, P. R., Davis, G. C., and Gri ng, B. F., "Contrast enhanced photolithography: application of photobleaching processes in microlithography," Journal of Imaging Science 30(2), 65-8 (1986).

[7] Kuebler, S. M., Braun, K. L., Zhou, W., Cammack, J. K., Yu, T., Ober, C. K., Marder, S. R., and Perry, J. W., "Design and application of high-sensitivity two-photon initiators for three-dimensional microfabrication," Journal of Photochemistry and Photobiology A: Chemistry 158(2-3), 163-170 (2003).

[8] Gelbart, D. and Karasyuk, V. A., "UV thermoresists: sub-100-nm imaging without proximity e ects," Proc. SPIE 3676(2), 786-793 (1999).

[9] Chapman, G. H., Tu, Y., and Peng, J., "Wavelength invariant Bi/In thermal resist as a Si anisotropic etch masking layer and direct-write photomask material," Proc. SPIE 5039, 472-483 (2003).

[10] Chapra, S. C. and Canale, R. P., [Numerical Methods For Engineers: With Software and Programming], McGraw-Hill, New York, 4th ed. (2002). 